\title{
36. DATA REPORT: COMPOSITION OF BASALTIC LAVAS FROM THE SEAWARD-DIPPING REFLECTOR SEQUENCE RECOVERED DURING DEEP SEA DRILLING PROJECT LEG 81 (HATTON BANK) ${ }^{1}$
}

\author{
James A. Brodie ${ }^{2}$ and J. Godfrey Fitton ${ }^{3}$
}

Deep Sea Drilling Project (DSDP) Leg 81 on Hatton Bank was the first successful attempt to determine the origin of seaward-dipping reflector sequences (SDRS), now known to be a characteristic feature of volcanic rifted margins. Basaltic lava flows cored at each of the four sites (552-555) established the volcanic origin of SDRS (Roberts, Schnitker, et al., 1984).

Hatton Bank, on the southwestern tip of Rockall Plateau, forms the southern limit of the North Atlantic SDRS, and the basaltic rocks recovered during Leg 81 represent volcanism distal to the axis of the ancestral Iceland plume. As such, these rocks provide useful reference material with which to compare basaltic lavas from other parts of the North Atlantic igneous province, especially other parts of the SDRS. The strongly depleted nature of these basalts has been noted by Joron et al. (1984), Richardson et al. (1984), and Merriman et al. (1988). All these authors comment on the similarity between the Hatton Bank basalt and normal mid-ocean-ridge basalt (N-MORB).

The purpose of this brief report is to present new X-ray fluorescence (XRF) spectrometric data for a suite of basaltic lavas from Hatton Bank, analyzed as part of a larger study on North Atlantic magmatism (Brodie, 1995). The data were obtained in Edinburgh, in the same laboratory and by essentially the same methods as those used for the analysis of Leg 152 samples (Fitton et al., Chap. 28, this volume) and the results are therefore directly comparable. The Hatton Bank samples were ground in a tungsten carbide mill, whereas those from Leg 152 were ground in agate, but we have no reason to believe that this will have affected any of the elements analyzed in this study. Grinding in tungsten carbide is known to contaminate samples with $\mathrm{W}, \mathrm{Co}$, and Ta, but we have not been able to detect any addition of $\mathrm{Nb}$. Great care was taken over the determination of the very low concentrations of $\mathrm{Nb}$, which are thought to be accurate to better than $\pm 0.2 \mathrm{ppm}$.

The new data (Table 1, and included on CD-ROM, back pocket, this volume) are used by Fitton et al. (Chap. 28, this volume) and Larsen et al. (Chap. 27, this volume) as a reference set for comparison with data from basaltic rocks recovered during Leg 152. Basaltic lavas forming the post-breakup SDRS at $63^{\circ} \mathrm{N}$ on the southeast Greenland Margin are broadly similar to the Hatton Bank basalts in their depletion in incompatible elements and in their unusually high Sc contents. However, basalts from the two sections of SDRS differ in detail. The N-MORB character of the Hatton Bank basalts is confirmed, but post-breakup lavas from $63^{\circ} \mathrm{N}$, closer to the center of the ancestral Iceland plume, are shown to resemble depleted Icelandic basalt.

${ }^{1}$ Saunders, A.D., Larsen, H.C., and Wise, S.W., Jr. (Eds.), 1998. Proc. ODP, Sci. Results, 152: College Station, TX (Ocean Drilling Program).

${ }^{2}$ Department of Earth Sciences, University of Cambridge, Bullard Laboratories, Maddingley Road, Cambridge CB2 3EZ, United Kingdom (Present address: Department of Geology, University of Bristol, Bristol BS8 1TR, United Kingdom). j.brodie@bristol.ac.uk

${ }^{3}$ Department of Geology and Geophysics, University of Edinburgh, West Mains Road, Edinburgh EH9 3JW, United Kingdom.

\section{REFERENCES}

Brodie, J., 1995. Early Tertiary magmatism in the North Atlantic [Ph.D. dissert.]. Cambridge Univ., UK.

Joron, J.L., Bougault, H., Maury, R.C., Bohn, M., and Desprairies, A., 1984. Strongly depleted tholeiites from the Rockall Plateau margin, North Atlantic: geochemistry and mineralogy. In Roberts, D.G., Schnitker, D., et al., Init. Repts. DSDP, 81: Washington (U.S. Govt. Printing Office), 783-794.

Merriman, R.J., Taylor, P.N., and Morton, A.C., 1988. Petrochemistry and isotope geochemistry of early Palaeogene basalts forming the dipping reflector sequence SW of Rockall Plateau, NE Atlantic. In Morton, A.C., and Parson, L.M. (Eds.), Early Tertiary Volcanism and the Opening of the NE Atlantic. Geol. Soc. Spec. Publ. London, 39:123-134.

Richardson, C., Oakley, P.J., and Cann, J.R., 1984. Trace and major element chemistry of basalts from Leg 81. In Roberts, D.G., and Schnitker, D., et al., Init. Repts. DSDP, 81: Washington (U.S. Govt. Printing Office), 795806.

Roberts, D.G., Schnitker, D., et al., 1984. Init. Repts. DSDP, 81: Washington (U.S. Govt. Printing Office).

Date of initial receipt: 8 November 1995

Date of acceptance: 23 May 1996

Ms 152SR-250 
Table 1. XRF analyses of volcanic rocks from Hatton Bank (DSDP Leg 81).

\begin{tabular}{|c|c|c|c|c|c|c|c|c|c|c|c|c|c|c|}
\hline Sample: & R55201 & R55202 & R55204A & R55204B & R55205 & R55206 & R55305 & R55308 & R55309 & R55310 & R55311 & R55312 & R55313 & R55314 \\
\hline Hole: & 552 & 552 & 552 & 552 & 552 & 552 & $553 \mathrm{~A}$ & $553 \mathrm{~A}$ & $553 \mathrm{~A}$ & $553 \mathrm{~A}$ & $553 \mathrm{~A}$ & $553 \mathrm{~A}$ & $553 \mathrm{~A}$ & $553 \mathrm{~A}$ \\
\hline Core: & 22 & 22 & 22 & 22 & 23 & 23 & 39 & 40 & 40 & 41 & 41 & 42 & 42 & 42 \\
\hline Section: & 1 & 1 & 2 & 2 & 1 & 2 & 2 & 3 & 4 & 1 & 2 & 1 & 2 & 3 \\
\hline Interval $(\mathrm{cm})$ : & $0-3$ & $34-36$ & $15-18$ & $41-43$ & $136-138$ & $9-12$ & $30-32$ & $101-104$ & $58-61$ & $55-59$ & $47-50$ & $92-95$ & $57-60$ & $91-94$ \\
\hline Piece: & 1 & 4 & 2 & $4 \mathrm{~A}$ & 1 & 1 & 3 & 1D & $2 \mathrm{C}$ & 9 & 7 & 12 & $6 \mathrm{~B}$ & 11 \\
\hline Depth (mbsf): & 284.00 & 284.34 & 285.65 & 285.91 & 289.86 & 290.09 & 513.80 & 525.51 & 526.58 & 531.55 & 532.97 & 541.42 & 542.57 & 544.41 \\
\hline $\mathrm{SiO}_{2}$ & 46.41 & 46.22 & 46.52 & 47.36 & 46.80 & 46.90 & 49.29 & 52.87 & 52.97 & 52.48 & 52.53 & 48.75 & 49.01 & 48.81 \\
\hline $\mathrm{Al}_{2} \mathrm{O}_{3}$ & 10.54 & 11.52 & 11.02 & 12.58 & 11.50 & 12.07 & 14.52 & 13.13 & 13.30 & 14.52 & 14.36 & 16.30 & 15.10 & 14.17 \\
\hline $\mathrm{Fe}_{2} \mathrm{O}_{3}{ }^{3}$ & 19.99 & 19.59 & 19.53 & 18.14 & 19.15 & 18.63 & 13.02 & 14.35 & 14.41 & 12.89 & 13.02 & 11.23 & 12.27 & 14.42 \\
\hline $\mathrm{MgO}$ & 10.60 & 9.02 & 8.88 & 6.68 & 8.28 & 7.70 & 7.07 & 5.68 & 5.67 & 5.94 & 5.70 & 7.50 & 7.67 & 7.05 \\
\hline $\mathrm{CaO}$ & 3.76 & 3.98 & 4.47 & 5.75 & 5.16 & 5.76 & 10.18 & 8.64 & 8.81 & 7.95 & 7.82 & 9.71 & 10.76 & 10.13 \\
\hline $\mathrm{Na}_{2} \mathrm{O}$ & 2.66 & 2.87 & 2.85 & 3.03 & 2.64 & 2.88 & 2.55 & 2.90 & 2.87 & 3.19 & 3.04 & 2.68 & 2.45 & 2.40 \\
\hline $\mathrm{K}_{2} \mathrm{O}$ & 0.11 & 0.13 & 0.14 & 0.21 & 0.15 & 0.16 & 0.24 & 0.09 & 0.10 & 0.08 & 0.09 & 0.04 & 0.04 & 0.06 \\
\hline $\mathrm{TiO}_{2}$ & 3.06 & 3.54 & 3.28 & 3.80 & 3.29 & 3.25 & 1.34 & 1.32 & 1.32 & 1.51 & 1.52 & 1.21 & 1.18 & 1.51 \\
\hline $\mathrm{MnO}$ & 0.14 & 0.14 & 0.11 & 0.12 & 0.11 & 0.12 & 0.23 & 0.34 & 0.29 & 0.23 & 0.27 & 0.23 & 0.24 & 0.28 \\
\hline $\mathrm{P}_{2} \mathrm{O}_{5}$ & 0.28 & 0.32 & 0.28 & 0.36 & 0.29 & 0.28 & 0.10 & 0.14 & 0.14 & 0.17 & 0.17 & 0.08 & 0.06 & 0.10 \\
\hline LOI & 2.59 & 2.71 & 2.87 & 1.99 & 2.69 & 2.02 & 1.12 & 0.52 & 0.48 & 1.37 & 1.43 & 2.29 & 1.51 & 1.48 \\
\hline Total & 100.13 & 100.04 & 99.95 & 100.02 & 100.06 & 99.77 & 99.65 & 99.98 & 100.36 & 100.33 & 99.94 & 100.02 & 100.29 & 100.40 \\
\hline $\mathrm{Nb}$ & 3.8 & 4.1 & 4.0 & 5.1 & 4.0 & 4.1 & 1.0 & 1.2 & 1.1 & 1.2 & 1.0 & 0.8 & 0.8 & 0.7 \\
\hline $\mathrm{Zr}$ & 181.2 & 191.1 & 181.2 & 235.0 & 188.9 & 183.9 & 66.4 & 107.2 & 103.6 & 114.2 & 115.9 & 55.0 & 54.9 & 52.4 \\
\hline $\mathrm{Y}$ & 66.7 & 68.1 & 66.2 & 83.9 & 69.3 & 66.8 & 31.1 & 43.0 & 42.3 & 39.7 & 38.5 & 24.4 & 18.8 & 26.4 \\
\hline $\mathrm{Sr}$ & 105.4 & 112.1 & 107.3 & 127.0 & 110.8 & 115.5 & 78.7 & 69.5 & 70.3 & 81.0 & 80.3 & 85.6 & 78.9 & 76.6 \\
\hline $\mathrm{Rb}$ & 0.4 & 0.6 & 0.4 & 1.6 & 0.6 & 1.0 & 6.3 & 0.3 & 1.0 & 0.7 & 0.4 & 0.1 & 0.0 & 0.8 \\
\hline $\mathrm{Zn}$ & 149.1 & 147.1 & 138.4 & 169.1 & 147.9 & 147.4 & 135.8 & 120.1 & 118.6 & 132.9 & 121.9 & 110.5 & 105.8 & 111.9 \\
\hline $\mathrm{Cu}$ & 239 & 265 & 277 & 272 & 265 & 271 & 75 & 235 & 82 & 120 & 76 & 369 & 220 & 176 \\
\hline $\mathrm{Ni}$ & 50 & 57 & 64 & 72 & 58 & 78 & 83 & 48 & 48 & 56 & 54 & 86 & 95 & 87 \\
\hline $\mathrm{Cr}$ & 103 & 148 & 122 & 127 & 149 & 185 & 129 & 55 & 55 & 80 & 82 & 221 & 185 & 185 \\
\hline V & 525 & 631 & 631 & 659 & 624 & 650 & 456 & 365 & 369 & 383 & 397 & 447 & 420 & 409 \\
\hline $\mathrm{Ba}$ & 12 & 21 & 14 & 22 & 13 & 16 & $<5$ & $<5$ & $<5$ & $<5$ & $<5$ & $<5$ & $<5$ & $<5$ \\
\hline $\mathrm{Sc}$ & 54 & 57 & 60 & 63 & 58 & 59 & 62 & 49 & 51 & 57 & 57 & 67 & 62 & 58 \\
\hline
\end{tabular}

Notes: Major elements in weight percent, trace elements in ppm. *Total $\mathrm{Fe}$ as $\mathrm{Fe}_{2} \mathrm{O}_{3}$.

Table 1 (continued).

\begin{tabular}{|c|c|c|c|c|c|c|c|c|c|c|c|c|c|c|}
\hline Sample: & R55315 & R55316 & R55317 & R55318 & R55319 & R55320 & R55321 & R55322 & R55323 & R55324 & R55325 & R55326 & R55327 & R55328 \\
\hline Hole: & $553 \mathrm{~A}$ & $553 \mathrm{~A}$ & 553A & $553 \mathrm{~A}$ & $553 \mathrm{~A}$ & 553A & $553 \mathrm{~A}$ & 553A & 553A & $553 \mathrm{~A}$ & 553A & $553 \mathrm{~A}$ & $553 \mathrm{~A}$ & $553 \mathrm{~A}$ \\
\hline Core: & 43 & 43 & 43 & 43 & 44 & 44 & 44 & 45 & 45 & 45 & 45 & 46 & 46 & 46 \\
\hline Section: & 2 & 3 & 4 & 5 & 1 & 2 & 3 & 3 & 4 & 5 & 6 & 1 & 2 & 3 \\
\hline Interval $(\mathrm{cm})$ : & $41-44$ & $106-109$ & $81-84$ & $97-100$ & $68-71$ & $87-90$ & $37-40$ & $14-17$ & $132-135$ & $131-134$ & $86-89$ & $86-89$ & $65-68$ & $113-116$ \\
\hline Piece: & 3 & $8 \mathrm{~A}$ & $1 \mathrm{G}$ & $1 \mathrm{~F}$ & $2 \mathrm{~A}$ & $2 \mathrm{~A}$ & $1 \mathrm{C}$ & 3 & $8 \mathrm{~F}$ & $4 \mathrm{C}$ & $2 \mathrm{E}$ & $1 \mathrm{E}$ & $2 \mathrm{~B}$ & $2 \mathrm{D}$ \\
\hline Depth (mbsf): & 551.91 & 554.06 & 555.31 & 556.97 & 560.18 & 561.87 & 562.87 & 565.14 & 567.82 & 569.31 & 570.36 & 572.36 & 573.65 & 575.63 \\
\hline $\mathrm{SiO}_{2}$ & 50.90 & 48.50 & 50.74 & 49.05 & 50.88 & 51.10 & 51.03 & 48.99 & 48.97 & 49.51 & 49.64 & 49.41 & 49.72 & 49.80 \\
\hline $\mathrm{Al}_{2} \mathrm{O}_{3}$ & 14.25 & 13.88 & 13.23 & 14.65 & 13.17 & 13.19 & 14.63 & 15.07 & 14.06 & 13.78 & 13.64 & 13.57 & 13.61 & 13.60 \\
\hline $\mathrm{Fe}_{2} \mathrm{O}_{3}{ }^{3}$ & 14.89 & 14.44 & 15.38 & 12.50 & 15.35 & 15.22 & 13.52 & 13.38 & 13.22 & 14.10 & 14.32 & 14.05 & 14.24 & 14.33 \\
\hline $\mathrm{MgO}$ & 5.87 & 6.79 & 6.06 & 7.53 & 6.13 & 6.19 & 6.02 & 7.57 & 7.13 & 7.55 & 7.45 & 7.67 & 7.77 & 7.69 \\
\hline $\mathrm{CaO}$ & 7.83 & 11.25 & 9.70 & 11.25 & 9.57 & 9.76 & 8.73 & 8.10 & 11.77 & 11.25 & 11.30 & 11.24 & 11.20 & 11.32 \\
\hline $\mathrm{Na}_{2} \mathrm{O}$ & 3.10 & 2.60 & 2.55 & 2.34 & 2.66 & 2.47 & 2.91 & 2.68 & 2.25 & 2.18 & 2.21 & 2.15 & 2.13 & 2.16 \\
\hline $\mathrm{K}_{2} \mathrm{O}$ & 0.17 & 0.31 & 0.07 & 0.04 & 0.07 & 0.06 & 0.07 & 0.41 & 0.04 & 0.04 & 0.05 & 0.04 & 0.05 & 0.04 \\
\hline $\mathrm{TiO}_{2}$ & 1.58 & 2.28 & 1.45 & 1.12 & 1.45 & 1.40 & 1.53 & 1.21 & 1.15 & 1.12 & 1.14 & 1.09 & 1.08 & 1.11 \\
\hline $\mathrm{MnO}$ & 0.18 & 0.21 & 0.28 & 0.23 & 0.29 & 0.28 & 0.27 & 0.15 & 0.30 & 0.25 & 0.25 & 0.27 & 0.26 & 0.25 \\
\hline $\mathrm{P}_{2} \mathrm{O}_{5}$ & 0.14 & 0.21 & 0.13 & 0.08 & 0.13 & 0.12 & 0.13 & 0.04 & 0.08 & 0.08 & 0.08 & 0.07 & 0.08 & 0.08 \\
\hline LOI & 1.38 & 0.22 & 0.60 & 1.22 & 0.34 & 0.37 & 1.13 & 2.53 & 1.06 & 0.52 & 0.26 & 0.15 & 0.32 & 0.15 \\
\hline Total & 100.29 & 100.70 & 100.19 & 100.02 & 100.02 & 100.16 & 99.96 & 100.13 & 100.03 & 100.38 & 100.33 & 99.71 & 100.45 & 100.53 \\
\hline $\mathrm{Nb}$ & 1.0 & 0.9 & 1.1 & 1.3 & 1.0 & 1.1 & 1.0 & 0.7 & 0.7 & 0.4 & 0.6 & 0.2 & 0.7 & 0.8 \\
\hline $\mathrm{Zr}$ & 102.3 & 95.6 & 95.2 & 101.1 & 92.6 & 93.2 & 95.4 & 55.2 & 57.0 & 54.2 & 54.9 & 52.3 & 51.0 & 53.6 \\
\hline$Y$ & 44.4 & 41.4 & 43.9 & 42.6 & 42.8 & 43.5 & 41.5 & 16.5 & 29.3 & 28.0 & 28.5 & 27.7 & 27.3 & 28.3 \\
\hline $\mathrm{Sr}$ & 79.0 & 68.5 & 69.9 & 74.7 & 69.5 & 69.5 & 78.4 & 79.3 & 74.3 & 69.4 & 69.6 & 69.0 & 68.4 & 69.0 \\
\hline $\mathrm{Rb}$ & 2.9 & 0.5 & 0.4 & 0.3 & 0.6 & 0.6 & 0.1 & 6.2 & 0.1 & 0.4 & 0.2 & 0.1 & 0.5 & 0.2 \\
\hline $\mathrm{Zn}$ & 162.5 & 126.4 & 132.8 & 131.6 & 124.8 & 127.3 & 126.7 & 186.2 & 106.9 & 104.5 & 108.2 & 102.3 & 100.2 & 103.3 \\
\hline $\mathrm{Cu}$ & 84 & 320 & 123 & 161 & 181 & 163 & 150 & 136 & 154 & 55 & 88 & 111 & 220 & 132 \\
\hline $\mathrm{Ni}$ & 58 & 61 & 59 & 59 & 59 & 61 & 62 & 77 & 80 & 81 & 79 & 79 & 79 & 79 \\
\hline $\mathrm{Cr}$ & 94 & 89 & 89 & 95 & 89 & 93 & 108 & 107 & 98 & 97 & 91 & 94 & 95 & 93 \\
\hline V & 493 & 436 & 435 & 471 & 434 & 418 & 481 & 421 & 419 & 404 & 397 & 387 & 386 & 389 \\
\hline $\mathrm{Ba}$ & $<5$ & $<5$ & $<5$ & $<5$ & $<5$ & $<5$ & $<5$ & $<5$ & $<5$ & $<5$ & $<5$ & $<5$ & $<5$ & $<5$ \\
\hline Sc & 62 & 52 & 54 & 60 & 54 & 55 & 66 & 65 & 60 & 56 & 55 & 56 & 55 & 57 \\
\hline
\end{tabular}


Table 1 (continued).

\begin{tabular}{|c|c|c|c|c|c|c|c|c|c|c|c|c|c|c|}
\hline Sample: & R55330 & R55331 & R55334 & R55335 & R55337 & R55338 & R55340 & R55341 & R55344 & R55345 & R55347 & R55349 & R55351 & R55352 \\
\hline Hole: & $553 \mathrm{~A}$ & 553A & $553 \mathrm{~A}$ & $553 \mathrm{~A}$ & 553A & $553 \mathrm{~A}$ & $553 \mathrm{~A}$ & $553 \mathrm{~A}$ & $553 \mathrm{~A}$ & $553 \mathrm{~A}$ & $553 \mathrm{~A}$ & $553 \mathrm{~A}$ & $553 \mathrm{~A}$ & $553 \mathrm{~A}$ \\
\hline Core: & 46 & 46 & 47 & 47 & 48 & 48 & 48 & 48 & 49 & 49 & 49 & 50 & 50 & 51 \\
\hline Section: & 5 & 5 & 3 & 4 & 2 & 3 & 5 & 6 & 3 & 4 & 6 & 1 & 3 & 1 \\
\hline Interval $(\mathrm{cm})$ : & $59-62$ & $138-141$ & $80-84$ & $76-79$ & $122-125$ & $133-136$ & $88-91$ & $107-110$ & $72-75$ & $108-111$ & $33-36$ & $71-75$ & $9-12$ & $85-88$ \\
\hline Piece: & $5 \mathrm{~A}$ & $6 \mathrm{H}$ & 2B & $5 \mathrm{~B}$ & 4 & 1B & $2 \mathrm{C}$ & $4 B$ & 1B & 1D & 1B & 11 & $1 \mathrm{~A}$ & $6 \mathrm{C}$ \\
\hline Depth (mbsf): & 578.09 & 578.88 & 584.30 & 585.76 & 590.22 & 591.83 & 594.38 & 596.07 & 600.22 & 602.08 & 604.33 & 606.21 & 608.59 & 615.35 \\
\hline $\mathrm{SiO}_{2}$ & 48.60 & 48.89 & 49.13 & 48.77 & 49.01 & 49.10 & 49.32 & 49.10 & 49.12 & 48.75 & 48.90 & 48.44 & 49.12 & 49.38 \\
\hline $\mathrm{Al}_{2} \mathrm{O}_{3}$ & 13.75 & 13.92 & 13.51 & 14.28 & 13.45 & 13.24 & 13.26 & 13.34 & 13.32 & 13.55 & 14.38 & 13.73 & 14.86 & 15.21 \\
\hline $\mathrm{Fe}_{2}^{2} \mathrm{O}_{3}^{*}$ & 16.09 & 15.49 & 15.23 & 14.03 & 15.28 & 15.57 & 15.64 & 15.32 & 15.67 & 15.08 & 13.79 & 13.94 & 12.27 & 11.58 \\
\hline $\mathrm{MgO}$ & 7.68 & 7.54 & 7.13 & 7.41 & 7.04 & 7.16 & 7.24 & 7.09 & 7.07 & 7.01 & 7.16 & 9.27 & 9.30 & 7.73 \\
\hline $\mathrm{CaO}$ & 8.57 & 9.39 & 10.01 & 8.70 & 10.70 & 10.54 & 10.64 & 10.67 & 10.81 & 10.88 & 10.47 & 9.12 & 9.46 & 11.14 \\
\hline $\mathrm{Na}_{2} \mathrm{O}$ & 2.63 & 2.55 & 2.50 & 2.83 & 2.28 & 2.23 & 2.18 & 2.23 & 2.25 & 2.49 & 2.48 & 2.30 & 2.42 & 2.34 \\
\hline $\mathrm{K}_{2} \mathrm{O}$ & 0.07 & 0.07 & 0.06 & 0.10 & 0.05 & 0.06 & 0.05 & 0.05 & 0.05 & 0.06 & 0.07 & 0.05 & 0.05 & 0.04 \\
\hline $\mathrm{TiO}_{2}$ & 1.50 & 1.49 & 1.49 & 1.67 & 1.44 & 1.43 & 1.40 & 1.41 & 1.41 & 1.45 & 1.53 & 0.96 & 1.01 & 1.09 \\
\hline $\mathrm{MnO}$ & 0.13 & 0.15 & 0.24 & 0.18 & 0.29 & 0.26 & 0.23 & 0.29 & 0.22 & 0.20 & 0.21 & 0.16 & 0.21 & 0.25 \\
\hline $\mathrm{P}_{2} \mathrm{O}_{5}$ & 0.11 & 0.11 & 0.11 & 0.14 & 0.12 & 0.11 & 0.11 & 0.11 & 0.11 & 0.11 & 0.11 & 0.03 & 0.07 & 0.08 \\
\hline LOI & 1.39 & 1.12 & 0.97 & 2.10 & 0.33 & 0.52 & 0.13 & 0.48 & 0.32 & 0.19 & 0.88 & 2.12 & 1.83 & 1.56 \\
\hline Total & 100.52 & 100.73 & 100.38 & 100.21 & 99.99 & 100.21 & 100.20 & 100.10 & 100.34 & 99.76 & 99.98 & 100.13 & 100.60 & 100.39 \\
\hline $\mathrm{Nb}$ & 0.7 & 0.7 & 0.9 & 1.1 & 0.6 & 0.8 & 0.7 & 0.7 & 1.0 & 0.9 & 0.8 & 0.6 & 0.6 & 0.4 \\
\hline $\mathrm{Zr}$ & 73.8 & 76.1 & 76.4 & 82.3 & 75.1 & 74.5 & 73.0 & 74.8 & 74.4 & 74.0 & 77.3 & 46.3 & 47.3 & 51.5 \\
\hline Y & 31.3 & 39.0 & 37.5 & 38.9 & 36.2 & 36.3 & 35.5 & 36.9 & 36.0 & 36.2 & 35.0 & 34.3 & 26.8 & 23.4 \\
\hline $\mathrm{Sr}$ & 80.4 & 78.8 & 78.5 & 86.1 & 74.0 & 73.1 & 71.1 & 72.5 & 70.5 & 74.5 & 79.0 & 68.8 & 71.8 & 78.0 \\
\hline $\mathrm{Rb}$ & 0.7 & 1.4 & 0.4 & 1.6 & 0.1 & 0.9 & 0.4 & 0.3 & 0.3 & -0.1 & 0.5 & 1.0 & 0.6 & 0.6 \\
\hline $\mathrm{Zn}$ & 109.4 & 119.5 & 119.9 & 135.8 & 120.0 & 121.7 & 118.9 & 124.1 & 121.4 & 116.6 & 117.6 & 80.3 & 88.2 & 101.9 \\
\hline $\mathrm{Cu}$ & 142 & 73 & 83 & 204 & 121 & 138 & 113 & 181 & 209 & 121 & 198 & 32 & 180 & 200 \\
\hline $\mathrm{Ni}$ & 74 & 71 & 70 & 73 & 83 & 82 & 81 & 83 & 81 & 79 & 86 & 93 & 96 & 91 \\
\hline $\mathrm{Cr}$ & 101 & 98 & 95 & 103 & 152 & 153 & 156 & 160 & 157 & 157 & 168 & 180 & 195 & 164 \\
\hline V & 455 & 464 & 464 & 520 & 427 & 445 & 426 & 444 & 440 & 436 & 474 & 353 & 385 & 412 \\
\hline $\mathrm{Ba}$ & $<5$ & $<5$ & $<5$ & $<5$ & $<5$ & $<5$ & $<5$ & $<5$ & $<5$ & $<5$ & $<5$ & $<5$ & $<5$ & $<5$ \\
\hline $\mathrm{Sc}$ & 61 & 59 & 55 & 64 & 54 & 53 & 53 & 54 & 56 & 54 & 62 & 56 & 63 & 62 \\
\hline
\end{tabular}

Table 1 (continued).

\begin{tabular}{|c|c|c|c|c|c|c|c|c|c|c|c|c|c|c|}
\hline Sample: & R55353 & R55355 & R55358 & R55359 & R55361 & R55364 & R55365 & R55366 & R55367 & R55369 & R55371 & R55372 & R55373 & R55375 \\
\hline Hole: & $553 \mathrm{~A}$ & $553 \mathrm{~A}$ & $553 \mathrm{~A}$ & $553 \mathrm{~A}$ & $553 \mathrm{~A}$ & $553 \mathrm{~A}$ & $553 \mathrm{~A}$ & $553 \mathrm{~A}$ & $553 \mathrm{~A}$ & $553 \mathrm{~A}$ & $553 \mathrm{~A}$ & $553 \mathrm{~A}$ & $553 \mathrm{~A}$ & $553 \mathrm{~A}$ \\
\hline Core: & 51 & 52 & 52 & 53 & 53 & 54 & 54 & 54 & 54 & 55 & 55 & 55 & 56 & 56 \\
\hline Section: & 2 & 1 & 4 & 1 & 3 & 2 & 3 & 4 & 5 & 3 & 5 & 6 & 1 & 3 \\
\hline Interval $(\mathrm{cm})$ : & $62-65$ & $52-55$ & $33-36$ & $81-84$ & $118-121$ & $62-65$ & $112-115$ & $95-98$ & $58-61$ & $117-120$ & $78-81$ & $50-54$ & $12-15$ & $87-90$ \\
\hline Piece: & $2 \mathrm{~B}$ & $2 \mathrm{~B}$ & $4 \mathrm{C}$ & $6 \mathrm{D}$ & 15 & 10 & 8D & $2 \mathrm{D}$ & 4 & 8 & $1 \mathrm{G}$ & 1B & 2 & 9 \\
\hline Depth (mbsf): & 616.62 & 618.52 & 622.83 & 624.31 & 627.68 & 634.62 & 636.62 & 637.95 & 639.08 & 645.67 & 648.28 & 649.50 & 650.62 & 654.37 \\
\hline $\mathrm{SiO}_{2}$ & 49.26 & 49.62 & 48.52 & 49.26 & 49.50 & 48.99 & 49.83 & 49.88 & 49.29 & 49.02 & 49.38 & 49.78 & 49.11 & 48.90 \\
\hline $\mathrm{Al}_{2} \mathrm{O}_{3}$ & 14.06 & 13.91 & 13.84 & 14.62 & 14.85 & 12.05 & 13.30 & 13.35 & 14.63 & 14.12 & 13.71 & 13.89 & 14.41 & 14.75 \\
\hline $\mathrm{Fe}_{2} \mathrm{O}_{3}{ }^{*}$ & 13.05 & 13.40 & 16.77 & 12.37 & 13.68 & 18.86 & 15.94 & 16.06 & 14.27 & 14.40 & 14.01 & 14.16 & 12.71 & 12.27 \\
\hline $\mathrm{MgO}$ & 7.76 & 8.01 & 7.80 & 7.88 & 7.03 & 8.19 & 6.79 & 6.91 & 7.09 & 7.38 & 7.61 & 7.30 & 8.26 & 9.49 \\
\hline $\mathrm{CaO}$ & 11.42 & 11.36 & 7.01 & 11.05 & 9.42 & 5.48 & 9.93 & 10.08 & 8.67 & 10.05 & 11.01 & 11.18 & 9.92 & 8.46 \\
\hline $\mathrm{Na}_{2} \mathrm{O}$ & 2.20 & 2.15 & 2.43 & 2.29 & 2.62 & 2.20 & 2.36 & 2.36 & 2.79 & 2.59 & 2.18 & 2.23 & 2.40 & 2.50 \\
\hline $\mathrm{K}_{2} \mathrm{O}$ & 0.04 & 0.04 & 0.47 & 0.04 & 0.04 & 0.68 & 0.04 & 0.04 & 0.07 & 0.04 & 0.03 & 0.04 & 0.04 & 0.04 \\
\hline $\mathrm{TiO}_{2}$ & 1.03 & 1.01 & 1.07 & 1.09 & 1.42 & 1.21 & 1.33 & 1.29 & 1.50 & 1.21 & 1.10 & 1.13 & 1.18 & 1.11 \\
\hline $\mathrm{MnO}$ & 0.25 & 0.29 & 0.09 & 0.24 & 0.32 & 0.10 & 0.34 & 0.32 & 0.24 & 0.23 & 0.36 & 0.32 & 0.25 & 0.18 \\
\hline $\mathrm{P}_{2} \mathrm{O}_{5}$ & 0.07 & 0.07 & 0.03 & 0.08 & 0.10 & 0.07 & 0.09 & 0.09 & 0.09 & 0.08 & 0.08 & 0.09 & 0.09 & 0.07 \\
\hline LOI & 0.97 & 0.55 & 1.96 & 1.29 & 1.31 & 2.61 & 0.53 & 0.16 & 1.62 & 1.09 & 0.74 & 0.24 & 1.66 & 2.46 \\
\hline Total & 100.11 & 100.41 & 99.98 & 100.20 & 100.29 & 100.44 & 100.48 & 100.54 & 100.25 & 100.22 & 100.21 & 100.35 & 100.03 & 100.22 \\
\hline $\mathrm{Nb}$ & 0.4 & 0.7 & 0.2 & 0.5 & 0.6 & 0.6 & 0.6 & 0.7 & 0.7 & 0.6 & 0.3 & 0.7 & 0.7 & 0.6 \\
\hline $\mathrm{Zr}$ & 49.5 & 48.5 & 50.4 & 50.6 & 65.1 & 55.1 & 64.1 & 62.7 & 67.7 & 57.2 & 53.4 & 56.8 & 54.9 & 50.6 \\
\hline $\mathrm{Y}$ & 25.9 & 26.1 & 17.1 & 25.8 & 33.8 & 21.4 & 35.4 & 33.5 & 37.0 & 29.3 & 28.7 & 29.3 & 27.7 & 18.8 \\
\hline $\mathrm{Sr}$ & 68.3 & 66.7 & 73.7 & 75.2 & 81.2 & 69.8 & 70.8 & 72.0 & 81.1 & 71.4 & 69.3 & 67.6 & 77.5 & 78.3 \\
\hline $\mathrm{Rb}$ & 0.4 & 0.1 & 7.1 & 0.0 & 0.6 & 9.0 & 0.3 & -0.1 & 0.6 & 0.0 & 0.6 & 0.7 & 0.4 & 0.3 \\
\hline $\mathrm{Zn}$ & 101.4 & 97.3 & 73.9 & 101.4 & 123.5 & 82.8 & 126.5 & 119.6 & 129.0 & 110.8 & 107.1 & 111.5 & 101.5 & 112.0 \\
\hline $\mathrm{Cu}$ & 186 & 182 & 94 & 188 & 96 & 191 & 124 & 206 & 199 & 71 & 176 & 137 & 399 & 317 \\
\hline $\mathrm{Ni}$ & 86 & 85 & 77 & 84 & 63 & 55 & 62 & 60 & 64 & 79 & 79 & 82 & 85 & 84 \\
\hline $\mathrm{Cr}$ & 150 & 147 & 121 & 132 & 90 & 63 & 69 & 66 & 76 & 129 & 127 & 127 & 138 & 140 \\
\hline V & 383 & 374 & 385 & 407 & 492 & 388 & 452 & 449 & 497 & 422 & 400 & 406 & 429 & 412 \\
\hline $\mathrm{Ba}$ & $<5$ & $<5$ & $<5$ & $<5$ & $<5$ & $<5$ & $<5$ & $<5$ & $<5$ & $<5$ & $<5$ & $<5$ & $<5$ & $<5$ \\
\hline $\mathrm{Sc}$ & 55 & 54 & 60 & 59 & 68 & 57 & 58 & 56 & 69 & 57 & 57 & 56 & 60 & 60 \\
\hline
\end{tabular}


Table 1 (continued).

\begin{tabular}{|c|c|c|c|c|c|c|c|c|c|c|c|c|c|c|}
\hline Sample: & R55378 & R55379 & R55380 & R55383 & R55401 & R55402 & R55403 & R55404 & R55405 & R55406 & R55501 & R55502 & R55503 & R55504 \\
\hline Hole: & $553 \mathrm{~A}$ & $553 \mathrm{~A}$ & $553 \mathrm{~A}$ & $553 \mathrm{~A}$ & $554 \mathrm{~A}$ & $554 \mathrm{~A}$ & $554 \mathrm{~A}$ & $554 \mathrm{~A}$ & $554 \mathrm{~A}$ & $554 \mathrm{~A}$ & 555 & 555 & 555 & 555 \\
\hline Core: & 58 & 58 & 58 & 59 & 7 & 7 & 7 & 7 & 9 & 14 & 68 & 68 & 69 & 69 \\
\hline Section: & 1 & 2 & 3 & 4 & 1 & 2 & 3 & 4 & 1 & 1 & 2 & 3 & 1 & 4 \\
\hline Interval $(\mathrm{cm})$ : & $54-57$ & $75-78$ & $48-51$ & $80-83$ & $33-36$ & $70-74$ & $57-60$ & $29-32$ & $110-114$ & $32-35$ & $141-144$ & $122-126$ & $108-111$ & $46-49$ \\
\hline Piece: & 4D & 9B & 6 & $6 \mathrm{~A}$ & $3 \mathrm{~A}$ & $5 \mathrm{~B}$ & 8 & $3 \mathrm{~B}$ & 9 & 4 & 7C & $12 \mathrm{~A}$ & $3 \mathrm{~L}$ & 4 \\
\hline Depth (mbsf): & 669.04 & 670.75 & 671.98 & 678.30 & 133.33 & 135.20 & 136.57 & 137.79 & 180.00 & 199.82 & 673.41 & 674.72 & 681.08 & 684.96 \\
\hline $\mathrm{SiO}_{2}$ & 48.98 & 48.56 & 49.03 & 49.28 & 50.17 & 50.56 & 50.84 & 50.56 & 48.61 & 50.45 & 48.68 & 49.12 & 49.34 & 49.43 \\
\hline $\mathrm{Al}_{2} \mathrm{O}_{3}$ & 14.49 & 14.64 & 15.57 & 14.36 & 13.87 & 13.81 & 13.74 & 13.70 & 14.58 & 14.27 & 14.43 & 14.49 & 14.12 & 14.10 \\
\hline $\mathrm{Fe}_{2} \mathrm{O}_{3}{ }^{*}$ & 12.48 & 11.71 & 11.16 & 12.54 & 13.22 & 13.51 & 13.33 & 13.54 & 13.90 & 12.60 & 12.90 & 12.94 & 13.55 & 13.82 \\
\hline $\mathrm{MgO}$ & 9.24 & 9.18 & 9.05 & 8.31 & 6.79 & 7.01 & 6.97 & 7.07 & 6.24 & 6.91 & 6.55 & 6.52 & 6.75 & 6.36 \\
\hline $\mathrm{CaO}$ & 9.96 & 10.33 & 10.11 & 11.73 & 11.65 & 11.65 & 11.64 & 11.59 & 11.44 & 11.51 & 11.04 & 11.05 & 10.75 & 11.28 \\
\hline $\mathrm{Na}_{2} \mathrm{O}$ & 2.24 & 2.20 & 2.44 & 1.96 & 2.35 & 2.29 & 2.25 & 2.32 & 2.44 & 2.27 & 2.64 & 2.67 & 2.57 & 2.60 \\
\hline $\mathrm{K}_{2} \mathrm{O}$ & 0.20 & 0.04 & 0.05 & 0.04 & 0.26 & 0.29 & 0.24 & 0.22 & 0.28 & 0.33 & 0.04 & 0.04 & 0.07 & 0.04 \\
\hline $\mathrm{TiO}_{2}$ & 1.00 & 0.98 & 1.03 & 0.94 & 1.04 & 1.04 & 1.03 & 1.03 & 1.18 & 1.16 & 1.71 & 1.70 & 1.64 & 1.67 \\
\hline $\mathrm{MnO}$ & 0.10 & 0.20 & 0.18 & 0.27 & 0.21 & 0.21 & 0.21 & 0.21 & 0.18 & 0.19 & 0.32 & 0.29 & 0.24 & 0.26 \\
\hline $\mathrm{P}_{2} \mathrm{O}_{5}$ & 0.08 & 0.07 & 0.06 & 0.07 & 0.11 & 0.08 & 0.08 & 0.07 & 0.10 & 0.08 & 0.14 & 0.13 & 0.13 & 0.13 \\
\hline LOI & 1.83 & 2.53 & 1.79 & 0.70 & 0.21 & -0.16 & -0.65 & -0.12 & 0.81 & 0.51 & 1.55 & 1.01 & 0.94 & 0.54 \\
\hline Total & 100.59 & 100.44 & 100.45 & 100.19 & 99.87 & 100.29 & 99.68 & 100.20 & 99.76 & 100.28 & 99.99 & 99.96 & 100.10 & 100.24 \\
\hline $\mathrm{Nb}$ & 0.5 & 0.4 & 0.6 & 0.5 & 0.8 & 1.0 & 0.9 & 0.9 & 1.3 & 1.2 & 1.4 & 1.4 & 1.4 & 1.3 \\
\hline $\mathrm{Zr}$ & 46.8 & 46.6 & 48.4 & 46.8 & 50.8 & 53.0 & 52.4 & 51.5 & 61.5 & 59.9 & 100.0 & 98.4 & 96.5 & 99.8 \\
\hline $\mathrm{Y}$ & 23.9 & 25.5 & 18.2 & 25.5 & 25.0 & 25.4 & 26.5 & 24.7 & 27.7 & 27.2 & 41.3 & 40.5 & 39.7 & 41.2 \\
\hline $\mathrm{Sr}$ & 73.4 & 71.9 & 76.5 & 64.1 & 82.5 & 82.4 & 82.4 & 79.7 & 84.6 & 82.1 & 111.3 & 109.5 & 106.2 & 107.0 \\
\hline $\mathrm{Rb}$ & 2.9 & 0.1 & 0.4 & 0.8 & 4.0 & 7.1 & 4.8 & 4.3 & 4.3 & 5.7 & 0.5 & 0.4 & 1.0 & 0.9 \\
\hline $\mathrm{Zn}$ & 73.5 & 91.4 & 99.7 & 88.6 & 108.8 & 106.0 & 98.0 & 105.2 & 115.8 & 104.4 & 105.2 & 109.1 & 105.4 & 142.0 \\
\hline $\mathrm{Cu}$ & 45 & 103 & 127 & 147 & 102 & 100 & 123 & 120 & 158 & 158 & 249 & 250 & 238 & 246 \\
\hline $\mathrm{Ni}$ & 107 & 112 & 114 & 110 & 61 & 58 & 61 & 58 & 80 & 98 & 94 & 92 & 92 & 90 \\
\hline $\mathrm{Cr}$ & 332 & 373 & 401 & 327 & 78 & 74 & 76 & 75 & 283 & 273 & 217 & 216 & 217 & 217 \\
\hline V & 362 & 362 & 382 & 339 & 373 & 357 & 360 & 374 & 401 & 377 & 447 & 451 & 434 & 432 \\
\hline $\mathrm{Ba}$ & $<5$ & $<5$ & $<5$ & $<5$ & $<5$ & $<5$ & $<5$ & $<5$ & $<5$ & $<5$ & $<5$ & $<5$ & 8 & $<5$ \\
\hline $\mathrm{Sc}$ & 56 & 58 & 58 & 52 & 60 & 56 & 60 & 60 & 58 & 57 & 54 & 57 & 50 & 55 \\
\hline
\end{tabular}

Table 1 (continued).

\begin{tabular}{|c|c|c|c|c|c|c|c|c|c|c|c|c|c|c|}
\hline Sample: & R55505 & R55506 & R55507 & R55508 & R55509 & R55510 & R55511 & R55512 & R55513 & R55514 & R55515 & R55516 & R55517 & R55518 \\
\hline Hole: & 555 & 555 & 555 & 555 & 555 & 555 & 555 & 555 & 555 & 555 & 555 & 555 & 555 & 555 \\
\hline Core: & 70 & 75 & 76 & 76 & 80 & 81 & 82 & 83 & 90 & 90 & 91 & 95 & 95 & 96 \\
\hline Section: & 1 & 2 & 1 & 4 & 3 & 3 & 1 & 1 & 2 & 4 & 1 & 1 & 3 & 2 \\
\hline Interval $(\mathrm{cm})$ : & $127-130$ & $72-75$ & $81-84$ & $87-90$ & $116-119$ & $88-91$ & $124-127$ & $80-83$ & $63-66$ & $53-56$ & $92-95$ & $100-103$ & $16-19$ & $27-30$ \\
\hline Piece: & $5 B$ & $5 \mathrm{D}$ & $2 \mathrm{C}$ & $3 \mathrm{~A}$ & & $2 \mathrm{G}$ & $5 \mathrm{C}$ & $1 \mathrm{E}$ & $1 \mathrm{~A}$ & $1 \mathrm{E}$ & 1D & $4 \mathrm{~A}$ & 2 & 3 \\
\hline Depth (mbsf): & 690.77 & 739.22 & 747.31 & 751.87 & 788.66 & 797.88 & 804.74 & 813.80 & 881.63 & 884.53 & 889.92 & 928.00 & 930.16 & 938.27 \\
\hline $\mathrm{SiO}_{2}$ & 48.62 & 48.38 & 48.82 & 48.71 & 49.25 & 49.18 & 49.11 & 48.87 & 49.10 & 49.30 & 48.62 & 49.21 & 49.14 & 49.10 \\
\hline $\mathrm{Al}_{2} \mathrm{O}_{3}$ & 15.70 & 14.99 & 13.98 & 14.35 & 12.82 & 13.58 & 13.70 & 13.85 & 13.26 & 13.28 & 14.03 & 13.98 & 14.07 & 13.97 \\
\hline $\mathrm{Fe}_{2} \mathrm{O}_{3}$ & 10.94 & 10.37 & 11.56 & 11.57 & 13.20 & 12.87 & 12.32 & 12.86 & 14.27 & 14.04 & 13.41 & 12.82 & 12.75 & 12.69 \\
\hline $\mathrm{MgO}$ & 8.18 & 8.08 & 8.04 & 7.93 & 7.97 & 8.09 & 8.34 & 7.28 & 7.00 & 6.91 & 7.96 & 7.74 & 7.73 & 7.79 \\
\hline $\mathrm{CaO}$ & 10.62 & 11.66 & 12.34 & 12.40 & 10.60 & 9.93 & 9.94 & 11.56 & 11.22 & 11.37 & 9.84 & 12.55 & 12.58 & 12.51 \\
\hline $\mathrm{Na}_{2} \mathrm{O}$ & 2.54 & 2.42 & 2.32 & 2.40 & 2.64 & 2.63 & 2.54 & 2.53 & 2.61 & 2.49 & 2.66 & 2.10 & 2.23 & 2.22 \\
\hline $\mathrm{K}_{2} \mathrm{O}$ & 0.03 & 0.03 & 0.19 & 0.02 & 0.50 & 0.77 & 0.70 & 0.04 & 0.07 & 0.05 & 0.08 & 0.10 & 0.06 & 0.08 \\
\hline $\mathrm{TiO}_{2}$ & 1.07 & 1.08 & 1.02 & 1.05 & 1.20 & 1.27 & 1.18 & 1.26 & 1.32 & 1.27 & 1.33 & 1.05 & 1.04 & 1.04 \\
\hline $\mathrm{MnO}$ & 0.26 & 0.27 & 0.23 & 0.28 & 0.33 & 0.22 & 0.23 & 0.27 & 0.24 & 0.22 & 0.24 & 0.21 & 0.21 & 0.20 \\
\hline $\mathrm{P}_{2} \mathrm{O}_{5}$ & 0.07 & 0.07 & 0.07 & 0.07 & 0.08 & 0.08 & 0.09 & 0.08 & 0.08 & 0.09 & 0.09 & 0.07 & 0.07 & 0.07 \\
\hline LOI & 2.06 & 2.58 & 1.53 & 1.58 & 1.49 & 1.62 & 1.90 & 1.51 & 1.17 & 1.08 & 1.72 & 0.51 & 0.28 & 0.48 \\
\hline Total & 100.08 & 99.93 & 100.09 & 100.36 & 100.07 & 100.25 & 100.06 & 100.10 & 100.34 & 100.10 & 99.98 & 100.34 & 100.16 & 100.15 \\
\hline $\mathrm{Nb}$ & 0.2 & 0.5 & 0.6 & 0.7 & 0.7 & 0.6 & 0.7 & 0.9 & 0.8 & 0.9 & 0.9 & 0.7 & 0.5 & 0.4 \\
\hline $\mathrm{Zr}$ & 51.8 & 50.7 & 47.8 & 49.7 & 59.9 & 60.5 & 60.8 & 61.9 & 64.9 & 69.5 & 66.0 & 51.2 & 51.4 & 50.6 \\
\hline $\mathrm{Y}$ & 23.4 & 26.7 & 28.6 & 27.6 & 31.2 & 33.7 & 32.1 & 30.3 & 36.1 & 36.9 & 36.1 & 29.1 & 27.9 & 29.4 \\
\hline $\mathrm{Sr}$ & 94.4 & 94.6 & 95.0 & 86.6 & 93.0 & 101.1 & 88.6 & 91.6 & 89.0 & 89.7 & 97.1 & 74.8 & 75.3 & 75.2 \\
\hline $\mathrm{Rb}$ & 0.9 & 0.5 & 1.6 & 0.4 & 6.3 & 7.6 & 6.6 & 0.2 & 0.7 & 0.3 & 1.7 & 3.2 & 1.8 & 2.8 \\
\hline $\mathrm{Zn}$ & 86.5 & 95.9 & 86.0 & 90.6 & 94.5 & 84.1 & 98.0 & 101.4 & 101.1 & 104.6 & 104.3 & 111.9 & 124.2 & 101.5 \\
\hline $\mathrm{Cu}$ & 183 & 198 & 187 & 192 & 168 & 175 & 180 & 214 & 179 & 170 & 182 & 189 & 189 & 186 \\
\hline $\mathrm{Ni}$ & 88 & 95 & 88 & 88 & 64 & 65 & 64 & 85 & 61 & 62 & 71 & 86 & 87 & 88 \\
\hline $\mathrm{Cr}$ & 216 & 380 & 351 & 343 & 99 & 123 & 125 & 160 & 95 & 98 & 104 & 327 & 333 & 348 \\
\hline V & 360 & 371 & 351 & 346 & 385 & 364 & 377 & 413 & 404 & 371 & 407 & 340 & 341 & 348 \\
\hline $\mathrm{Ba}$ & $<5$ & $<5$ & $<5$ & $<5$ & 41 & 45 & 21 & $<5$ & $<5$ & $<5$ & 6 & 10 & $<5$ & 6 \\
\hline $\mathrm{Sc}$ & 56 & 62 & 56 & 55 & 51 & 54 & 52 & 57 & 52 & 54 & 61 & 55 & 55 & 56 \\
\hline
\end{tabular}


Table 1 (continued).

\begin{tabular}{|c|c|c|c|c|c|c|c|c|c|}
\hline Sample: & R55519 & R55520 & R55521 & R55522 & R55523 & R55524 & R55525 & R55526 & R55527 \\
\hline Hole: & 555 & 555 & 555 & 555 & 555 & 555 & 555 & 555 & 555 \\
\hline Core: & 96 & 96 & 97 & 97 & 97 & 98 & 98 & 98 & 98 \\
\hline Section: & 4 & 6 & 1 & 3 & 5 & 1 & 3 & 5 & 6 \\
\hline Interval $(\mathrm{cm})$ : & $81-85$ & $35-39$ & $81-84$ & $70-73$ & $102-105$ & $55-58$ & 104-107 & $75-78$ & $83-86$ \\
\hline Piece: & $3 \mathrm{~A}$ & 3 & 3B & $4 \mathrm{~A}$ & $2 \mathrm{D}$ & $1 \mathrm{H}$ & $3 \mathrm{G}$ & $4 \mathrm{~A}$ & 5 \\
\hline Depth (mbsf): & 941.81 & 944.35 & 946.81 & 949.70 & 953.02 & 955.55 & 959.04 & 961.75 & 963.33 \\
\hline $\mathrm{SiO}_{2}$ & 49.03 & 48.86 & 48.34 & 49.30 & 49.01 & 48.88 & 49.07 & 48.88 & 49.12 \\
\hline $\mathrm{Al}_{2} \mathrm{O}_{3}$ & 14.01 & 14.12 & 13.87 & 14.15 & 14.11 & 14.30 & 14.14 & 14.13 & 14.09 \\
\hline $\mathrm{Fe}_{2} \mathrm{O}_{3}{ }^{*}$ & 12.47 & 12.19 & 12.90 & 12.44 & 12.43 & 12.19 & 12.43 & 12.22 & 12.45 \\
\hline $\mathrm{MgO}$ & 7.87 & 7.85 & 7.71 & 7.85 & 7.84 & 7.85 & 8.03 & 8.08 & 8.03 \\
\hline $\mathrm{CaO}$ & 12.60 & 12.53 & 12.36 & 12.81 & 12.57 & 12.66 & 12.64 & 12.50 & 12.56 \\
\hline $\mathrm{Na}_{2} \mathrm{O}$ & 2.21 & 2.20 & 2.12 & 2.24 & 2.31 & 2.17 & 2.19 & 2.17 & 2.12 \\
\hline $\mathrm{K}_{2} \mathrm{O}$ & 0.06 & 0.04 & 0.04 & 0.04 & 0.05 & 0.04 & 0.05 & 0.05 & 0.04 \\
\hline $\mathrm{TiO}_{2}$ & 1.00 & 1.02 & 1.12 & 1.00 & 1.00 & 1.00 & 1.00 & 1.02 & 1.00 \\
\hline $\mathrm{MnO}$ & 0.20 & 0.21 & 0.21 & 0.21 & 0.20 & 0.19 & 0.20 & 0.20 & 0.20 \\
\hline $\mathrm{P}_{2} \mathrm{O}_{5}$ & 0.06 & 0.06 & 0.07 & 0.07 & 0.07 & 0.06 & 0.07 & 0.07 & 0.06 \\
\hline LOI & 0.58 & 1.09 & 0.84 & 0.23 & 0.69 & 0.85 & 0.49 & 0.61 & 0.45 \\
\hline Total & 100.09 & 100.17 & 99.58 & 100.34 & 100.28 & 100.20 & 100.30 & 99.92 & 100.12 \\
\hline $\mathrm{Nb}$ & 0.6 & 0.5 & 1.0 & 0.4 & 0.7 & 0.8 & 0.4 & 0.5 & 0.8 \\
\hline $\mathrm{Zr}$ & 48.6 & 47.3 & 47.9 & 48.7 & 49.2 & 46.6 & 48.5 & 50.4 & 49.9 \\
\hline $\mathrm{Y}$ & 27.9 & 27.5 & 28.0 & 28.0 & 28.3 & 28.1 & 27.1 & 27.9 & 28.6 \\
\hline $\mathrm{Sr}$ & 76.1 & 78.2 & 77.5 & 77.0 & 76.5 & 76.1 & 74.5 & 76.9 & 75.0 \\
\hline $\mathrm{Rb}$ & 1.5 & 0.7 & 0.7 & 0.9 & 1.5 & 0.3 & 1.1 & 1.1 & 1.8 \\
\hline $\mathrm{Zn}$ & 104.2 & 90.1 & 100.1 & 88.9 & 85.0 & 81.6 & 93.7 & 86.0 & 84.2 \\
\hline $\mathrm{Cu}$ & 188 & 186 & 178 & 191 & 177 & 175 & 177 & 184 & 181 \\
\hline $\mathrm{Ni}$ & 88 & 91 & 90 & 86 & 93 & 95 & 93 & 91 & 92 \\
\hline $\mathrm{Cr}$ & 351 & 355 & 347 & 306 & 397 & 411 & 397 & 382 & 388 \\
\hline $\mathrm{V}$ & 345 & 350 & 377 & 332 & 335 & 332 & 329 & 338 & 336 \\
\hline $\mathrm{Ba}$ & 6 & $<5$ & $<5$ & $<5$ & $<5$ & $<5$ & $<5$ & $<5$ & $<5$ \\
\hline $\mathrm{Sc}$ & 58 & 55 & 56 & 55 & 55 & 55 & 57 & 57 & 55 \\
\hline
\end{tabular}

